\title{
28 Research Suare \\ Investigation for Enhancement of 5-hydroxymethylfurfural extraction into 2, 5-furandicarboxylic acid: an Ab initio study
}

\section{Amir Jalalinejad}

Ca'Foscari University of Venice: Universita Ca' Foscari

Payam Ghorbannezhad ( $\sim$ p_ghorbannezhad@sbu.ac.ir)

Shahid Beheshti University https://orcid.org/0000-0002-6146-8964

Behnam Dehbandi

Islamic Azad University

Imtiaz Ali

King Abdulaziz University

\section{Research Article}

Keywords: 5-hydroxymethylfurfural (HMF), 2,5-furandicarboxylic acid (FDCA), COSMO-RS model, density function theory (DFT)

Posted Date: February 4th, 2022

DOI: https://doi.org/10.21203/rs.3.rs-1286967/v1

License: (c) (i) This work is licensed under a Creative Commons Attribution 4.0 International License. Read Full License 


\section{Abstract}

Furandicarboxylic acid (FDCA) is recognized as a valuable product of hydroxymethylfurfural (HMF) derived from cellulosic materials that could find future bioplastic application if a feasible separation process is developed. To find a commercially available solvent that can selectivity separate FDCA and HMF as well as the downstream process was supported by Py-GC-MS experiments with density functional theory (DFT). Evaluation of the sigma potential and sigma surface analysis showed that benzene and ethyl acetate have better extraction and selectivity of HMF, whereas FDCA indicated ideal behavior in DMF and DMSO solvents, where the hydrophobicity is changed by improving the hydrogenbonding interaction between them. The up-down selection of classes of solvents based on the experimental data found by GC-MS revealed that polar molecular solvents (ethanol-water) are more compatible with carboxylic acids and alcohol compounds, while n-hexane is a desirable solvent for phenolic compounds. It is discovered that levoglucosan retains a significant fraction of water compared to other solvents that need to be considered for further economic and environmental analyses under the multifaceted framework of biomass-derived products.

\section{Introduction}

Recently, bioplastics have become promising feedstocks for value-added products, which have the potential to replace fossil fuel-based petrochemicals. Petrochemicals pose environmental and climate-related challenges to our society. Environmental concerns increase when chemical manufacturers expand their product portfolio and increase production due to the continuously increasing demands for polymeric materials. More than $8000 \mathrm{Mt}$ of plastics have been consumed annually, roughly $80 \%$ of which have ended up in landfills and oceans (Geyer et al. 2017). This is because as consumers, we are becoming more addicted to single-use disposable plastics. The U.S. Department of Energy (DOE) predicted that plastic manufacturers will consume $20 \%$ of all petroleum materials with a share of $15 \%$ annual global carbon emissions by the year 2050 (Perlack et al. 2011). The global warning that arises from plastic production, consumption, and waste management requires renewable chemical strategies (Erickson and Winters 2012; Zheng and Suh 2019).

Biorefinery is recognized as a valuable route of using biomass as a feedstock that can reduce our dependence on fossil fuels (Stuart and El-Halwagi 2012). Biomass is an abundant feedstock with great potential to produce biofuels, biochemical, and bioproducts (Ghorbannezhad et al. 2020; Bilal et al. 2021). Today, more than 100 billion tons of biomass are available around the world, most of which end up as waste due to ineffective technologies (Dutta et al. 2014; Searle and Malins 2015). Biomass can completely boost the bioeconomy by rebuilding fuel and chemical manufacturing processes. Lignocellulosic biomass is made up of carbohydrate polymers such as cellulose (40-50\%) and hemicellulose (20-40\%) and aromatic polymers such as lignin (20-30\%). These chemical components are the main building blocks of many biochemical platforms (Figure 1).

5-hydroxymethylfurfural (HMF) is recognized as a high-value building block compound of cellulose that can be transformed into a variety of value-added chemicals (Kuster 1990; Rosatella et al. 2011; Davidson et al. 2021). HMF is recognized as the "top 10" chemical platform in the circular bioeconomy by DOE. It is synthesized by the dehydration of mono and polysaccharides present in lignocellulosic biomass. Furandicarboxylic acid (FDCA) is one of the most valuable biochemicals that can be derived from HMF by biological or chemical oxidation (Arikan et al. 2021). During conversion, the oxidation of the aldehyde of HMF produces 5-hydroxymethyl-2-furan carboxylic acid, which is an intermediate compound. Further oxidation of 5-hydroxymethyl-2-furan carboxylic acid produces furan carboxylic acid (FFCA), which finally turns into FDCA. The schematic of cellulose conversion is shown in Figure 2.

Therefore, one of the most cost-effective FDCA production routes could be the direct conversion of biomass into desired products on a commercial scale (Hwang et al. 2020; Arikan et al. 2021). In this regard, condensation reactions

Page $2 / 15$ 
under acidic aqueous conditions must be suppressed by substituting with an organic solvent during the rehydration process (Bonner et al. 1960). Solvents are vital to obtain a higher yield of HMF and its derivatives. Currently, direct dehydration of glucose to produce HMF and its derivatives is limited by an inefficient separation process (Cai et al. 2013; Hou et al. 2017; Motagamwala et al. 2019; Bello Ould-Amer et al. 2020; Zunita et al. 2021). FDCA has shown low solubility in water and the problem of deactivation during the direct conversion of glucose to HMF in water as a solvent (Zhang et al. 2018; Esteban et al. 2020). The weak separation efficiency of short-chain solvents led to high volumetric consumption of organic solvents and salts to make a biphasic system. However, selecting an efficient solvent for purification and separation of products is a new research area for the development of biorefinery processes. Methyl isobutyl ketone (MIBK), tetrahydrofuran (THF), and dimethyl sulfoxide (DMSO) have been used as moderate polar and high dielectric solvents to promote the dehydration reaction for the production of HMF (Weingarten et al. 2014). The authors indicated that the solvents increased the dehydration reaction but decreased the polymerization of HMF. Some studies revealed that the biphasic system aims to improve the HMF yield by in situ extraction of HMF with an organic phase. A higher partition coefficient of HMF can lead to increased productivity and make the process cost-effective (Blumenthal et al. 2016). The computational method applies a heuristic approach that estimates the activity coefficient to identify appropriate solvents. The combination of quantum mechanics and statistical mechanics presents multifunctional models to better describe molecular thermodynamics. Unlike empirical models such as UNIFAC, ASOG, etc., the conductor-like screening model for real solvents (COSMO-RS) can explain intermolecular interactions (Klamt 2011; Balchandani and Singh 2021). An ensemble of pairwise intermolecular interactions in COSMO-RS provides a significant deviation in phase behavior, leading to crucial insights into different solvent classes (Eckert and Klamt 2018). Density function theory (DFT) can be combined with COSMO-RS to optimize models (Momany and Schnupf 2014; Wang et al. 2020). This work aims to investigate and predict the thermodynamic properties of cellulose derivatives obtained from biomass. Five samples, including HMF, DFF, HMFCA, FDCA, and FFCA, were studied. Flash points, boiling points, Henry's constant, activity coefficients, vapor-liquid equilibrium (VLE) and liquid-liquid equilibrium (LLE) were obtained for various solvents and different dielectric constants. The products were identified and predicted via thermodynamic properties that have not been reported before.

\section{Materials And Methods}

The experimental pyrolysis of biomass was performed in Rx-300 TR, a tandem micropyrolyzer system from Frontier Laboratories, Japan. The system is classified into two parts: a) pyrolysis reactor with a steady amount of biomass of 1-

$2 \mathrm{mg}$ and b) catalyst bed reactor. Hence, you do not use the catalysts; you can set the reactor temperature as the same as the pyrolysis reactor temperature. The milled biomass samples were placed in a stainless steel cup to drop into the first reactor. A neutral carrier gas such as helium or nitrogen ( $1 \mathrm{ml} / \mathrm{min}$ ) transfers the vaporized biomass compound from the thermal degradation reactor to the catalyst bed reactor. A temperature of $500^{\circ} \mathrm{C}$ is fitted by a programmable temperature control to maintain a constant process condition. The hot vapor (syngas) is retained in the first reactor for less than 2 seconds and then goes into the catalyst bed reactor, which is finally detected by GC-MS interfaced with the pyrolysis system. The percentage area peak of each compound could be determined to estimate the yield of products individually.

\section{Computational Methods and Theory}

COSMO-RS is the conductor-like screening model for real solvation that uses quantum chemistry (QC) to predict the thermodynamic properties of molecules (Klamt 1995; Marsh 2006). The molecules are embedded in a virtual conductor based on the polarization charge densities of the solute and solvent molecules. 
DFT can be combined with the COSMO as it is available in most quantum chemical programs. The structure of the biomass used in this study was drawn manually using the Gauss view software, which was further optimized with the Gaussian 09 program (Keith and Millam 2016). From the optimized structure, COSMO, sigma profile potential, and frequency files were generated. With the level of theory, Becke's three-parameter functional for exchange was combined with the nonlocal correlation potential of the Lee-Yang-Parr functional and the DGTZVP basis set (Godbout et al. 1992; Sosa et al. 1992).

One of the important COSMO-RS calculations is to estimate the sigma profile of each sample studied. The specific interactions of each of the molecules in the aqueous phase were defined in COSMO-RS. The quantum mechanical characterization of solvents, such as electrostatic interactions and hydrogen bonding, local interactions of surface segments, is calculated and screened by the charge density profile $\sigma$ and stored in the COSMO-RS database. The segment interaction of individual solvents determined the efficiency and solubility of the compositions via statistical thermodynamic calculations for the interacting surfaces. The free energies and chemical potentials of the molecules in pure and mixed solvents are due to the efficiency of solubility and separation for each composition.

In this work, the COSMO-RS calculations were performed with the COSMOtherm (Cosmologic GmbH, Leverkusen, Germany) program with BP_TZVPD_18 (Klamt et al. 2016). The geometries and conformations used for the solutes and solvents were generated and handled in the COSMO-RS calculations as described before (Eckert and Klamt 2018). Using the COSMOtherm program, the thermodynamic properties of the extracted chemical compounds can be predicted, such as flashpoints, boiling point, density, activity coefficient, $\sigma$ surface, $\sigma$ potential, liquid vapor equilibrium (VLE), liquid-liquid equilibrium (LLE), vapor pressure, Henry's law coefficient and hexane-water partition coefficients at different solvents and various temperatures and pressures. In COSMO, the $\sigma$ profile of molecule X (Equation 1) and the chemical potential of a surface segment with screening charge density (Equation 2) were obtained.

$p^{X_{i}}(\sigma)=\frac{n_{i}(\sigma)}{n_{i}}=\frac{A_{i}(\sigma)}{A_{i}}$ Equation 1

where

$A_{i}(\sigma)$ is the segment surface area that has charge density $\sigma$

$A_{i}$ is the area of the whole surface cavity rooted in the medium.

$\mu_{S}(\sigma)=-\frac{R T}{a_{e f f}} \ln \left[\int p_{S}\left(\sigma^{\prime}\right) \exp \left\{\frac{a_{e f f}}{R T}\left[\mu_{S}\left(\sigma^{\prime}\right)-e\left(\sigma, \sigma^{\prime}\right)\right]\right\} d \sigma^{\prime}\right]$ Equation 2

where

$\mu_{s}\left(\sigma^{\prime}\right)$ is the chemical potential of a surface segment

$\sigma$ is the polarity of the surface under study.

Table1. Properties, energy efficacy, and toxicity of studied solvent 


\begin{tabular}{|c|c|c|c|c|c|c|c|c|}
\hline \multirow[t]{2}{*}{ Solvents } & \multicolumn{4}{|l|}{ Properties } & \multicolumn{4}{|c|}{ Energy efficiency } \\
\hline & $\begin{array}{l}\text { Molecular } \\
\text { weight } \\
\text { (g/mol) }\end{array}$ & $\begin{array}{l}\text { Viscosity } \\
(\mathrm{m} / \mathrm{s})\end{array}$ & $\log ^{-}$ & $\begin{array}{l}\text { Flash } \\
\text { point, } \\
{ }^{\circ} \mathrm{C}\end{array}$ & $\begin{array}{l}\text { Boiling } \\
\text { point, } \\
{ }^{\circ} \mathrm{C}\end{array}$ & $\begin{array}{l}\text { SH vap } \\
\text { (kJ/mol) }\end{array}$ & $\begin{array}{l}\text { Cp liq } \\
\left(\mathrm{J} \cdot \mathrm{kg}^{-1} \mathrm{~K}^{-1}\right)\end{array}$ & $\begin{array}{l}\text { Energy } \\
\text { evaporation } \\
(\mathrm{kJ} / \mathrm{mol})\end{array}$ \\
\hline Water & 18.01 & 1.0016 & -0.65 & N/A & 100 & 40.65 & 4185.5 & 40.6 \\
\hline Ethanol & 46.07 & 0.983 & -0.24 & 12 & 78 & 38.56 & 2.57 & 38.5 \\
\hline Methanol & 32.04 & 0.543 & -0.76 & 11 & 65 & 35.21 & 2.53 & 35.2 \\
\hline DMSO & 78.13 & 2 & -1.37 & 88.88 & 189 & 52.9 & 149.40 & 52,5 \\
\hline DMF & 73.09 & 0.92 & -1 & 60 & 153 & 56.7 & 146.05 & 46.7 \\
\hline Benzene & 78.11 & 0.603 & 2 & -11 & 80 & 30.72 & 133 & 33.8 \\
\hline $\begin{array}{l}\text { Ethyl- } \\
\text { acetate }\end{array}$ & 88.11 & 0.426 & 0.71 & -4.44 & 77 & 31.94 & 161.47 & 31,9 \\
\hline $\mathrm{CCl}_{4}$ & 153.82 & 0.965 & 2.8 & 982 & 77 & 29.82 & 133.0 & 30 \\
\hline hexane & 86.18 & 0.3 & 3.764 & -7 & 69 & 28.85 & 265.2 & 28.8 \\
\hline
\end{tabular}

\section{Results And Discussions Sigma Potential Analysis}

The $\delta$-potential describes the possible interaction of compounds with solvents according to polarity and hydrogen bonding. Molecular interactions between the solvents and HMF play a vital role in HMF solubility. The shape, size, and initial components of the molecule are essential for molecular interaction. Caloric profiles provide important information to predict molecular interactions in the fluid phase. The hydrogen bond acceptor, hydrogen bond donor, and non-polar area associated with red, blue, and green, respectively, are shown in Figure 3. For $\delta$-surface, the negative screening charge density represented the positive polarity. In general, acceptor hydrogen bonds have a negative charge density, whereas the donor hydrogen shows a positive charge density. For example, there are negative and positive sides to $n$-hexane. The negative side corresponds to hydrogens, whereas the positive side corresponds to carbons. In general, $n$-hexane shows a polar alkane characterization when the $\delta$-potential in near zero.

\section{Chemical compositions of solvents}

Several chemical compositions were obtained by GC-MS of biomass. The concentrated aqueous solution of cellulose enhances the generation of HMF. Water as a solvent resulted in a higher HMF and levulinic acid converted to 2-MTHMF, butanols and acid. Table 2 shows the chemical composition of cellulose in different solvents identified by GC-MS. The results showed that the DMF exhibits lower performance compared to ethyl acetate and hexane in water. More acid and esters (aliphatic compounds) were separated by ethanol-water solvents, whereas more aromatic compounds could be extracted by hexane and benzene as solvents. It is assumed that the low solubility of hydrocarbons in water is due to the reduction of the polarity differences. Typically, polar solvents enhance HMF and carboxylic acid extraction by $20 \%$. Cellulose is anticipated to reduce the solubility of phenolic compounds in water and affect the overall process separation. 
Table 2

Chemical compositions of solvent extraction by GC-MS

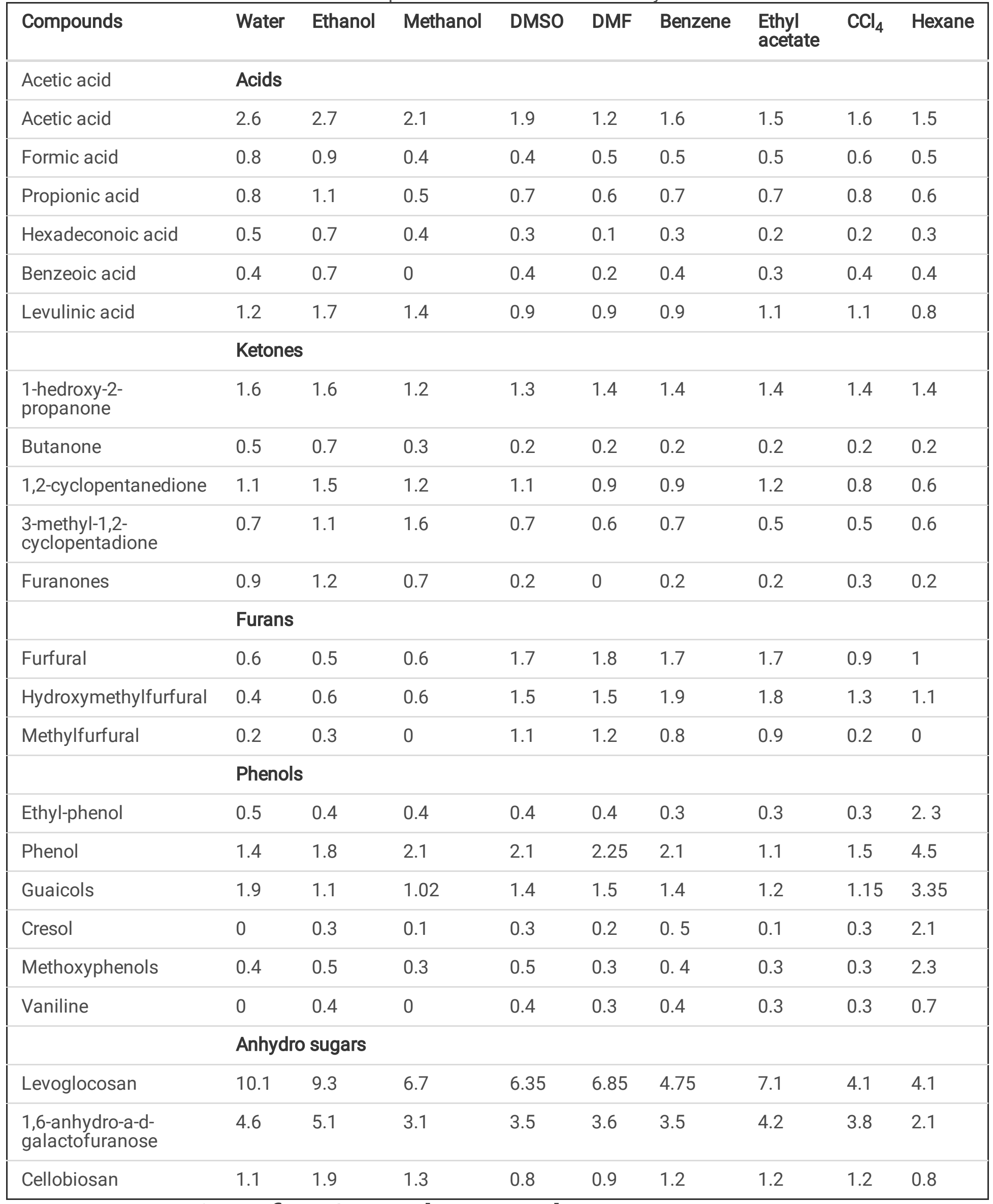


The free energy derivative validated the simulation of the interaction of FDCA separation in water and $\mathrm{n}$-hexane that corresponds to the process. The interaction of FDCA separation in $n$-hexane showed a Gibbs free energy value of +0.8 kcal.mol ${ }^{-1}$, whereas the same purification process in water is $-0.9 \mathrm{kcal}^{\mathrm{mol}}{ }^{-1}$ (Figure 4). However, n-hexane can be placed in the middle of the solvents, which theoretically could dissolve all compounds equally. Gibb's free energy is positive for $n$-hexane, illustrating low solubility limits for all concentration levels. The solubility of water in the organic phase of FDCA is lower compared to that of n-hexane. Thus, the Gibbs free energy presents a highly negative area in the water.

On the other hand, Gibbs energy of mixing is positive for an HMF-water system for the entire concentration range due to its very low mutual solubility limits. HMF-water is partially miscible, the solubility of water in the organic phase is higher as compared to the aqueous solubility. So the Excess Enthalpy of the mixing plot shows the negative region in the HMF region as shown in Figure 5. The HMF-water shows a maximum miscibility gap of a mole fraction of 0.9. Moreover, the Gibbs energy and excess enthalpy of mixing are negative for an FDCA-water system for the entire concentration range due to its mutual solubility limits. FDCA-water is partially miscible, the solubility of water in the organic phase is higher as compared to the aqueous solubility. Therefore, the Gibbs energy and excess enthalpy of the mixing plot shows the negative region in middle the FDCA- water region as shown in figure 4 (b).

The excess energies for HMF at solvent water / hexane at room temperature $(298.15 \mathrm{~K})$ is shown in figure 5. The figure is illustrated the calculation of excess properties of Gex and Hex respectively. HMF-water at system mixture are positive values (Gex energy of $\sim 0.2 \mathrm{~kJ} / \mathrm{mol}$ at $x 1=0.7$ ). Hexane for system mixture is minimum of $-0.05 \mathrm{~kJ} / \mathrm{mol}$ at $x 1=0.8$. In other hand HMF-hexane have the value of Gex energy of $\sim 0.6 \mathrm{~kJ} / \mathrm{mol}$ at $\mathrm{x} 1=0.6$ and Hex with a minimum of 0.7 $\mathrm{kJ} / \mathrm{mol}$ at $\mathrm{x} 1=0.65$.

\section{Activity Coefficients:}

For the calculation of activity coefficients on mole fraction scale for each solute (HMF/FDCA) in an infinite dilution state at COSMO-RS and COSMO SAC methods, the mole fraction of the solute is set to zero in the composition of the solution, and the reference state is the pure solute. Cosmotherm calculates the natural logarithmic values of the activity coefficient using the pseudo-chemical potentials of the compound both in its pure form and fully dissolved in a solvent.

It can be observed that the activity coefficient for the components HMF, FDCA gets very high for the case of infinite dilution at solvent hexane higher than solvent h20. This means that the activity coefficients and intermolecular interactions of Different solutes in solvents are very much dependent on the chemical structure of solute HMF and FDCA.

This non-ideality is attributed to the degree of dissociation/reaction of the solute, to the solute-solvent interactions such as complex ion formation, and to the solute-solute interactions such as ion pairing. An activity coefficient incorporates the particle interactions into a single term that modifies the formal concentration to give an estimate of the effective concentration, or activity, of each ion. The partition coefficients $(\log P)$ of infinitely diluted solutes in a mixture of two immiscible solvents can be calculated with Partition Coefficients (LogP). We used for calculation of ethanol/water, benzene/water, ether/water, and hexane/Water partition coefficients. In the case of partly miscible liquids, like the ethanol-rich phase of ethanol and water, both components have nonzero mole fractions. The preset also gives a value for the molar volume quotient of the two solvents. 
Table 3

The partition coefficient (LOG P) of mixture solvents

\begin{tabular}{|llllll|}
\hline Compound & ethanol/Water & Benzene/Water & Hexane/Water & Ether/Water \\
\hline HMF & -0.8337 & -1.9507 & -3.5269 & 0.1151 \\
\hline FDCA & 0.4527 & -3.4778 & -5.1710 & 2.9823 \\
\hline
\end{tabular}

\section{COSMO-RS Prediction Optimized by DFT}

The sigma potential determines the pseudochemical potential of the molecular surface. The chemical potential of the surface segment was obtained by thermodynamics of molecular interactions. Using COSMO-RS theory, one can calculate the thermodynamic properties of fluids from the 3D polarized charge distribution of individual molecules ( $\sigma$ surface) in terms of $\sigma$-profile (2D histogram). Thus, valuable information regarding the polarity of a compound and its interaction with other surrounding molecules in the fluid media can be deduced from these $\sigma$-profile histograms. Figure 6 shows $P_{X}(\sigma)$; the charge density of these compounds in the polarized field $(\sigma)$. The following criteria can be applied to divide the field into three regions: For $\sigma>+0.0082 \mathrm{e} / \AA^{-2}$ is the hydrogen bond (HB) acceptor region, the hydrogen bond $(\mathrm{HB})$ donor region has $\sigma<-0.0082 \mathrm{e} / \AA^{-2}$, and the nonpolar region between $-0.0082<\sigma<+0.0082 \mathrm{e} / \AA^{-2}$. The sigma profile of ethanol and methanol exhibited a positive potential for the polar surface segment $\left(\delta>0.05\right.$ e $\left.\AA^{-2}\right)$ implying an unfavorable solvation for HMF extraction. It is manifested that ethanol and methanol are miscible with water. It revealed that the HMF is a strong hydrogen bond donor because of the higher profile area that it favors with water interaction. The hydroxylmethyl group of HMF is the hydrogen-bonding donor from aqueous media. On the contrary, the benzene and ethyl acetate solvents show a descending sigma potential sinking below $-0.15 \mathrm{kcal} \mathrm{mol}^{-1} \AA^{-2}$ for the surface charge density at 0.05 e $\AA^{-2}$, where efficient HMF separation took place (Figure 6).

The sigma potential profiles suggest DMF and DMSO as the alternative and most promising solvents for FDCA extraction. The hydrophobicity region at $\delta=0$ reflected the potential solubility difference of the solvents with water. Benzene and ethyl acetate enable direct bonding to the $\mathrm{OH}$ group, resulting in an increase in the nonpolar alkyl group and thus of the hydrophobicity. On the other hand, DMF and DMSO replace the hydrophilic environment to improve the selectivity and overall yield of FCDA (Figure 7). In fact, the low mutual solubility of DMF and DMSO makes them not eligible to interact on hydrogen bonding. Thus, DMF and DMSO exhibited a lower charge density in hydrogen bonding, which results in less interaction with water and better fractionation of the organic phase. Thus, nonpolar organic and hydrocarbon compounds with $\pi-\pi$ bond are more favorable with DMF and DMSO solvents because of fewer interactions with the hydrogen bond. Nevertheless, it is interesting to note that there are clear differences in the interactions of solvents with HMF and FDCA.

Comparison of $\sigma$-profile derived from HMF and FDCA shows that the charge density of the HMF and FDCA compounds can be found in the nonpolar region of $\sigma$-profile, due to its heterocyclic ring (green in $\sigma$-surface). The functional groups (carboxylate, hydroxyl methyl) contain negative charge (red color in $\sigma$-surface) which is shifted towards the positive pole of the field. In FDCA, these peaks were found at higher positive polarity, showing a stronger HB acceptor character of the carboxylate group rather than the hydroxyl methyl group in the homologous HMF. Thus, the different profiles from $\sigma$-surface are contributed independently of each atom related to its charge density, as well as the descriptor of each constituent in the solvents. Finally, with stronger HB donor and HB acceptor groups, COSMO-RS describes FDCA as more polar structure than those of the homolog HMF. Consequently, the results of this study aimed to develop a framework of models that could predict the quantum-chemical properties of solvents to screen the interactions between them. It is purely a prediction approach of COSMO-RS optimized by the DFT methodology. On the other hand, it provides an efficient final product that is rich in HMF and FDCA and approves the emerging biobased molecules for 
commercializing green processes. Therefore, the efficient solvent design approach would be highly reliable with experimental data in the applicability domain for green and sustainable bioplastic manufacturing processes.

\section{Conclusions}

This study aims to evaluate the efficiency of different solvents for the desirable extraction of HMF and FDCA for future bioplastic industries. A multiscale COSMO-RS model on a desirable solvent system was optimized by DFT methodology. Evaluation of the sigma potential and sigma surface analysis showed that benzene and ethyl acetate have better extraction and selectivity to HMF. Moreover, FDCA indicated ideal behavior in DMF and DMSO solvents. GCMS data revealed that the polar molecular solvent (ethanol-water) extracted more carboxylic acids and alcohols, while n-hexane lies in the middle of the solvents for phenolic compounds. It is worth noting that the higher aromatics in HMF promote the Diels-Alder reaction as well as the dehydration reaction. Thus, a promising route for the generation of FDCA from HMF could be through the use of strong concentrations of cellulose. In addition to that, DMF and DMSO improve the hydrogen bonding interaction between FDCA and solvent molecules, and thus lead to efficient separation. However, the strategy employed in this study could be considered as a computational prediction to select a favorable solvent with efficient bioplastic manufacturing processes.

\section{Declarations}

\section{Acknowledgments}

This study was supported partially by department of biorefinery engineering at Shahid Beheshti University, Iran (award number: 397081).

\section{Conflict of interest}

The authors declare that they have no known competing financial interests or personal relationships that could have appeared to influence the work reported in this paper.

\section{References}

1. Arikan EB, Bouchareb EM, Bouchareb R et al (2021) Innovative Technologies Adopted for the Production of Bioplastics at Industrial Level. In: Bioplastics for Sustainable Development. Springer, pp 83-102

2. Balchandani S, Singh R (2021) Thermodynamic analysis using COSMO-RS studies of reversible ionic liquid 3aminopropyl triethoxysilane blended with amine activators for CO2 absorption. J Mol Liq 324:114713

3. Bello Ould-Amer S, Méndez Trelles P, Rodil Rodríguez E et al (2020) Towards improving the sustainability of bioplastics: Process modelling and life cycle assessment of two separation routes for 2, 5-furandicarboxylic acid

4. Bilal M, Vilar DS, Eguiluz KIB et al (2021) Biochemical conversion of lignocellulosic waste into renewable energy. Advanced Technology for the Conversion of Waste into Fuels and Chemicals. Elsevier, pp 147-171

5. Blumenthal LC, Jens CM, Ulbrich J et al (2016) Systematic identification of solvents optimal for the extraction of 5hydroxymethylfurfural from aqueous reactive solutions. ACS Sustain Chem Eng 4:228-235

6. Bonner TG, Bourne EJ, Ruszkiewicz M (1960) 160. The iodine-catalysed conversion of sucrose into 5-hydroxymethylfurfuraldehyde.J Chem Soc787-791

7. Cai CM, Zhang T, Kumar R, Wyman CE (2013) THF co-solvent enhances hydrocarbon fuel precursor yields from lignocellulosic biomass. Green Chem 15:3140-3145 
8. Davidson MG, Elgie S, Parsons S, Young TJ (2021) Production of HMF, FDCA and their derived products: a review of life cycle assessment (LCA) and techno-economic analysis (TEA) studies. Green Chem

9. Dutta S, Bhaumik A, Wu KC-W (2014) Hierarchically porous carbon derived from polymers and biomass: effect of interconnected pores on energy applications. Energy Environ Sci 7:3574-3592

10. Eckert F, Klamt A (2018) COSMOtherm, version 18.0. 0. Cosmol GmbH CoKG Leverkusen, Ger

11. Erickson B, Winters $P$ (2012) Perspective on opportunities in industrial biotechnology in renewable chemicals. Biotechnol J 7:176-185

12. Esteban J, Vorholt AJ, Leitner W (2020) An overview of the biphasic dehydration of sugars to 5hydroxymethylfurfural and furfural: a rational selection of solvents using COSMO-RS and selection guides. Green Chem 22:2097-2128

13. Geyer R, Jambeck JR, Law KL (2017) Production, use, and fate of all plastics ever made. Sci Adv 3:e1700782e1700782

14. Ghorbannezhad P, Park S, Onwudili JA (2020) Co-pyrolysis of biomass and plastic waste over zeolite- and sodiumbased catalysts for enhanced yields of hydrocarbon products. Waste Manag 102:909-918. https://doi.org/10.1016/j.wasman.2019.12.006

15. Godbout N, Salahub DR, Andzelm J, Wimmer E (1992) Optimization of Gaussian-type basis sets for local spin density functional calculations. Part I. Boron through neon, optimization technique and validation. Can J Chem 70:560-571

16. Hou Q, Li W, Zhen M et al (2017) An ionic liquid-organic solvent biphasic system for efficient production of 5hydroxymethylfurfural from carbohydrates at high concentrations. RSC Adv 7:47288-47296

17. Hwang K-R, Jeon W, Lee SY et al (2020) Sustainable bioplastics: Recent progress in the production of bio-building blocks for the bio-based next-generation polymer PEF. Chem Eng J 390:124636

18. Keith TA, Millam JM (2016) GaussView, Version 6.1, Roy Dennington. Semichem Inc

19. Klamt A (2011) The COSMO and COSMO-RS solvation models. Wiley Interdiscip Rev Comput Mol Sci 1:699-709

20. Klamt A (1995) Conductor-like screening model for real solvents: a new approach to the quantitative calculation of solvation phenomena. J Phys Chem 99:2224-2235

21. Klamt A, Eckert F, Reinisch J, Wichmann K (2016) Prediction of cyclohexane-water distribution coefficients with COSMO-RS on the SAMPL5 data set. J Comput Aided Mol Des 30:959-967

22. Kuster BFM (1990) 5-Hydroxymethylfurfural (HMF). A review focussing on its manufacture. Starch-Stärke 42:314321

23. Marsh KN, Klamt A (2006) Elsevier: Amsterdam, The Netherlands, 2005. 246 pp. \$ US 165. ISBN 0-444-51994-7

24. Momany F, Schnupf U (2014) DFT optimization and DFT-MD studies of glucose, ten explicit water molecules enclosed by an implicit solvent, COSMO. Comput Theor Chem 1029:57-67

25. Motagamwala AH, Huang K, Maravelias CT, Dumesic JA (2019) Solvent system for effective near-term production of hydroxymethylfurfural (HMF) with potential for long-term process improvement. Energy Environ Sci 12:22122222

26. Perlack RD, Eaton LM, Turhollow AF Jr et al (2011) US billion-ton update:. biomass supply for a bioenergy and bioproducts industry

27. Rosatella AA, Simeonov SP, Frade RFM, Afonso CAM (2011) 5-Hydroxymethylfurfural (HMF) as a building block platform: Biological properties, synthesis and synthetic applications. Green Chem 13:754-793

28. Searle S, Malins C (2015) A reassessment of global bioenergy potential in 2050. Gcb Bioenergy 7:328-336 
29. Sosa C, Andzelm J, Elkin BC et al (1992) A local density functional study of the structure and vibrational frequencies of molecular transition-metal compounds. J Phys Chem 96:6630-6636

30. Stuart PR, El-Halwagi MM (2012) Integrated biorefineries: design, analysis, and optimization. CRC press

31. Wang Z, Bhattacharyya S, Vlachos DG (2020) Solvent selection for biphasic extraction of 5-hydroxymethylfurfural via multiscale modeling and experiments. Green Chem 22:8699-8712

32. Weingarten R, Rodriguez-Beuerman A, Cao F et al (2014) Selective conversion of cellulose to hydroxymethylfurfural in polar aprotic solvents. ChemCatChem 6:2229-2234

33. Zhang Y, Guo X, Tang P, Xu J (2018) Solubility of 2, 5-Furandicarboxylic Acid in Eight Pure Solvents and Two Binary Solvent Systems at 313.15-363.15 K. J Chem Eng Data 63:1316-1324

34. Zheng J, Suh S (2019) Strategies to reduce the global carbon footprint of plastics. Nat Clim Chang 9:374-378

35. Zunita M, Wahyuningrum D, Bundjali B et al (2021) Conversion of Glucose to 5-Hydroxymethylfurfural, Levulinic Acid, and Formic Acid in 1, 3-Dibutyl-2-(2-butoxyphenyl)-4, 5-diphenylimidazolium lodide-Based lonic Liquid. Appl Sci 11:989

\section{Figures}

\section{Lignocellulosic Feedstock Biorefinary}

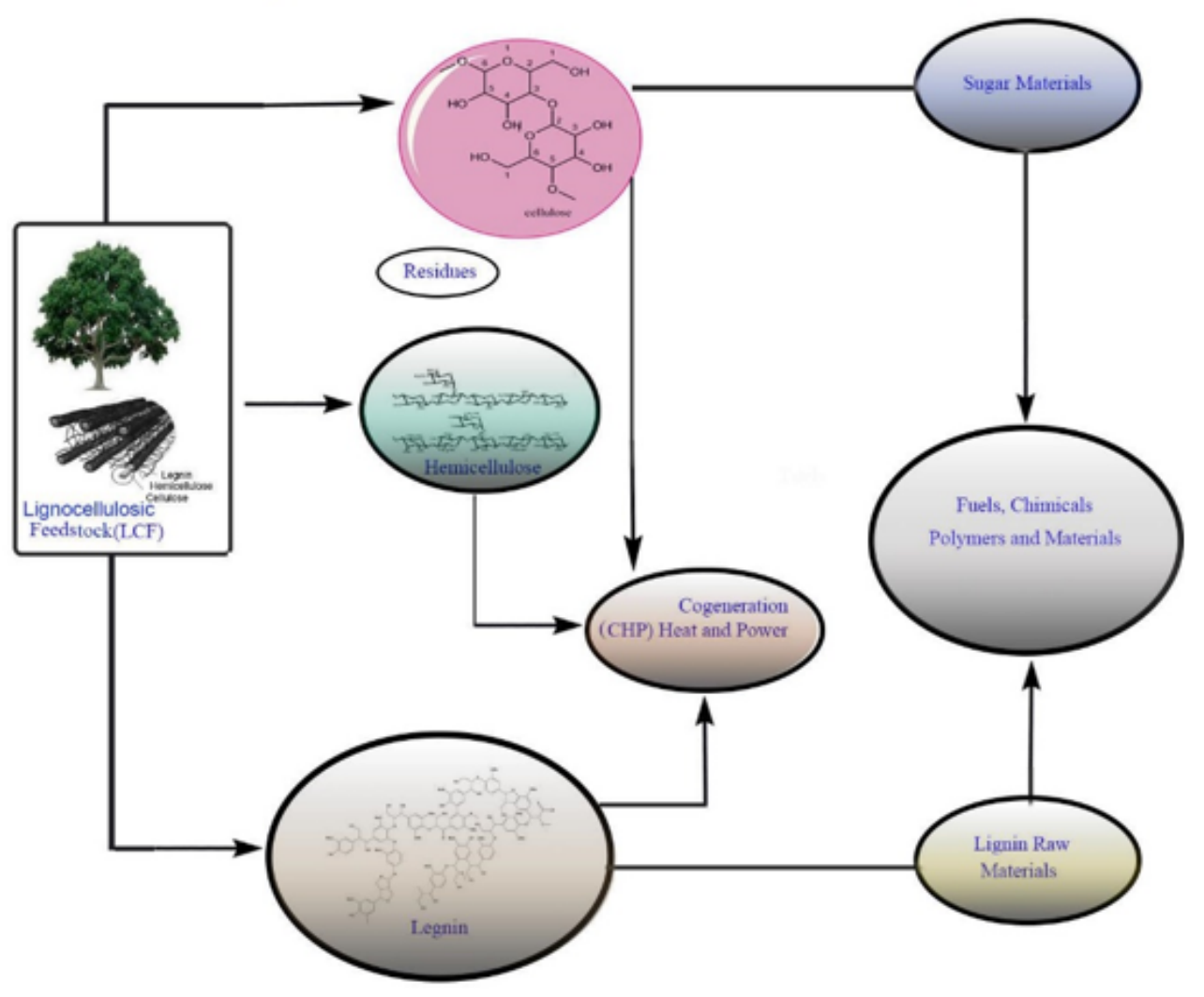

Figure 1

Pathways of value-added products from lignocellulosic components 


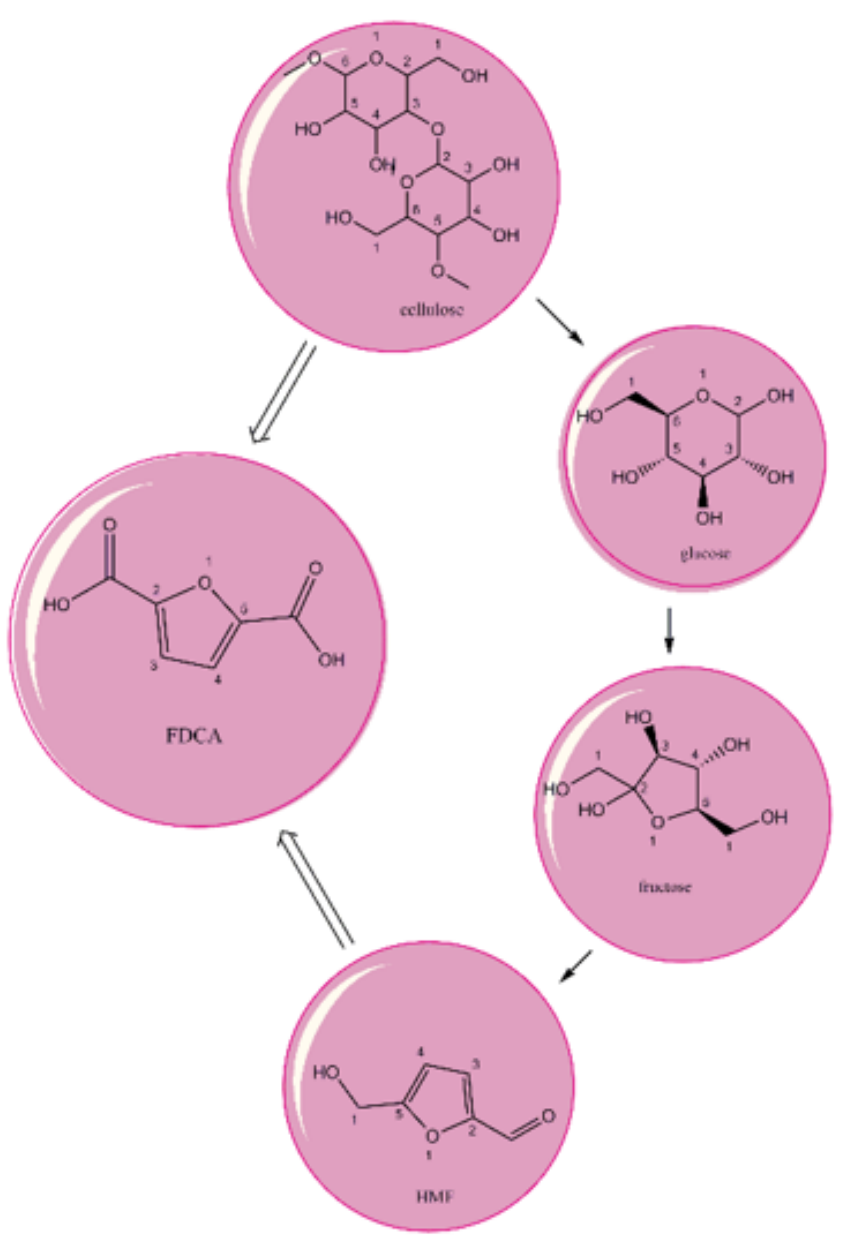

Figure 2

Overview of FDCA production pathways from cellulose

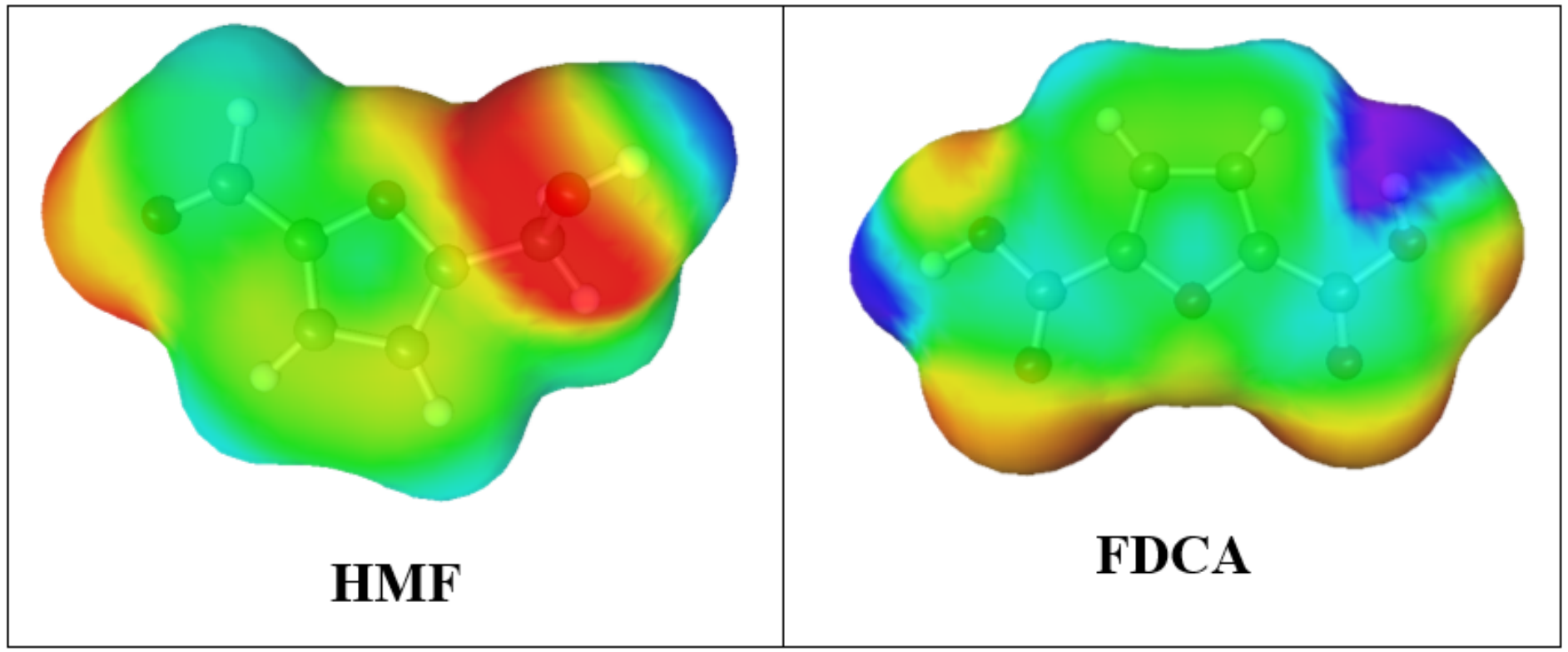

Figure 3 
Sigma surface of HMF and FDCA
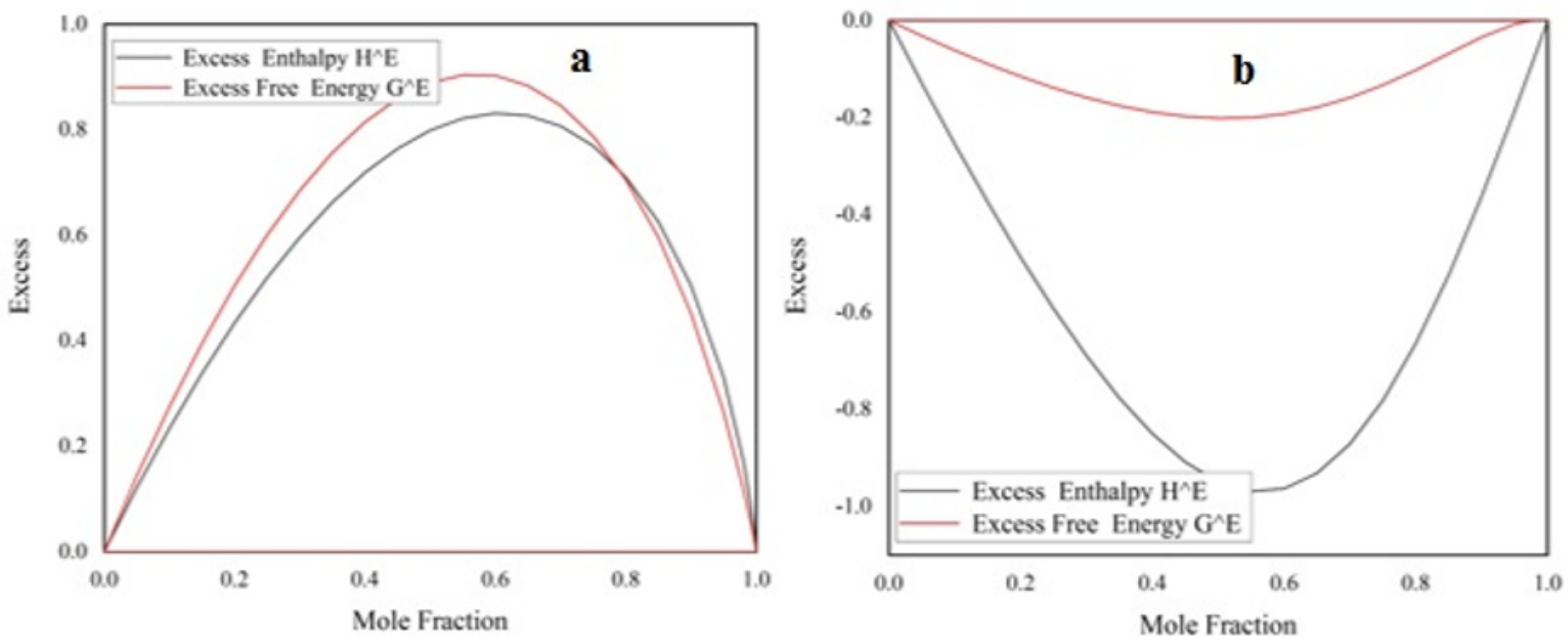

Figure 4

Gibbs tangent plane diagram for FDCA solvents (a: $n$-hexane; b: water)
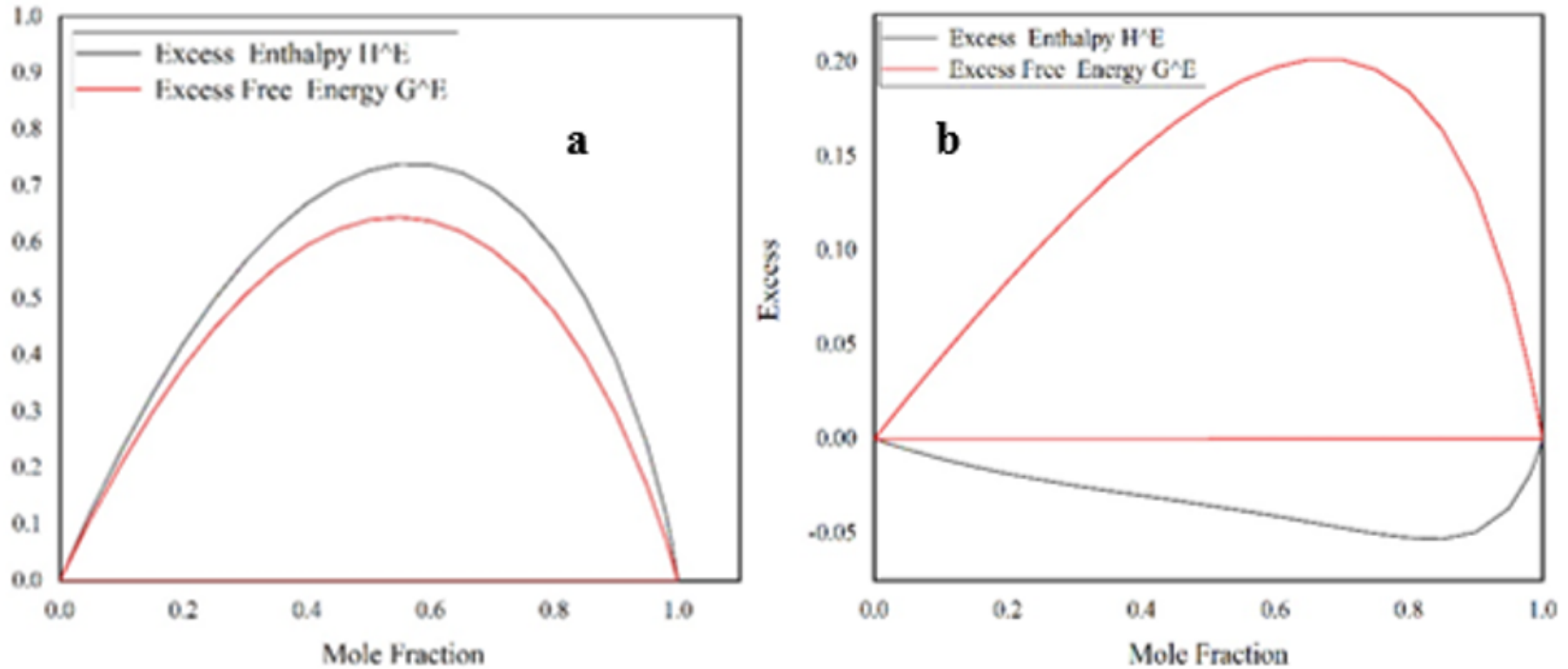

Figure 5

Gibb's tangent plane diagram for the HMF solvents (a: n-hexane; b: water) 


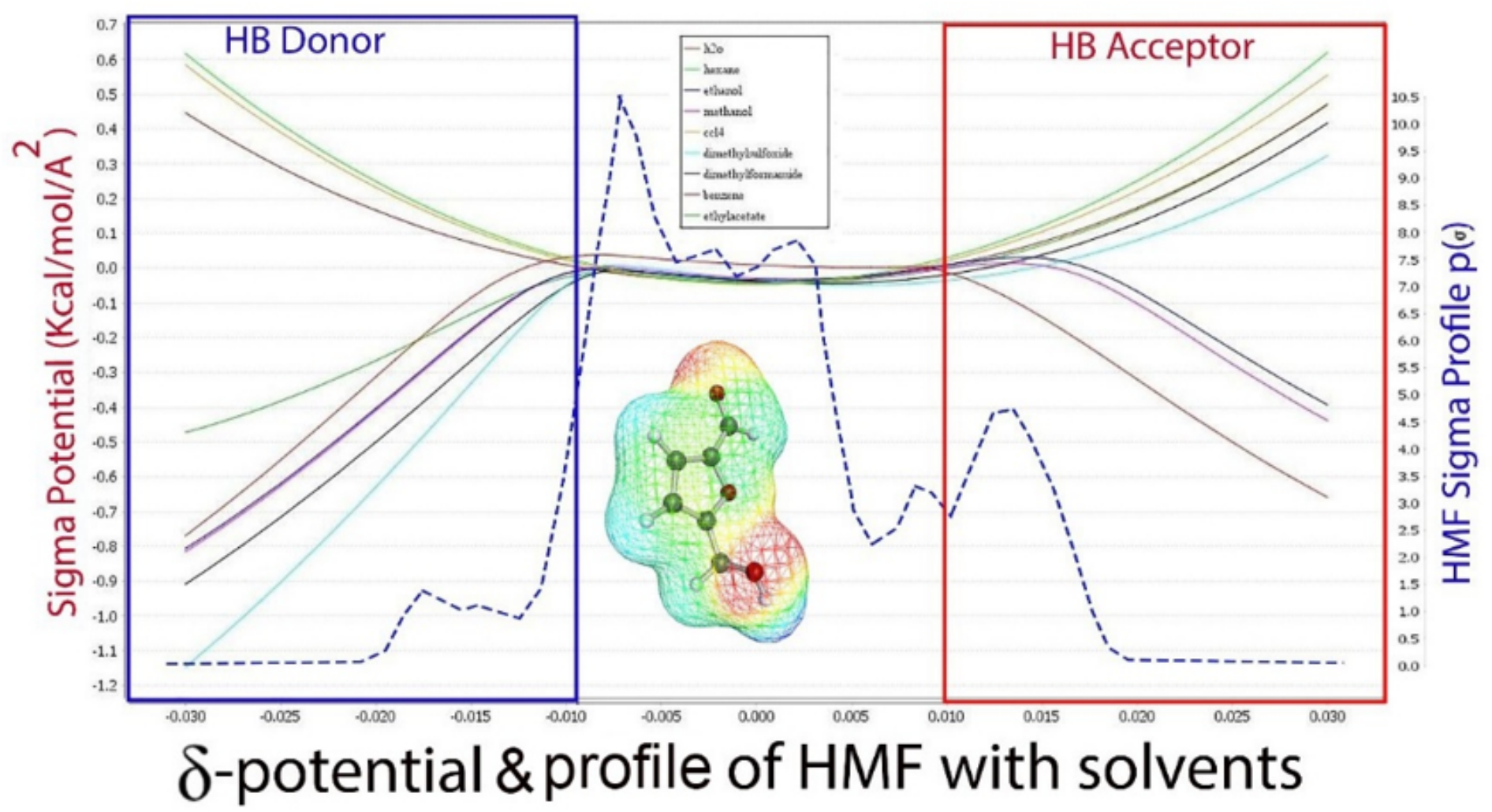

Figure 6

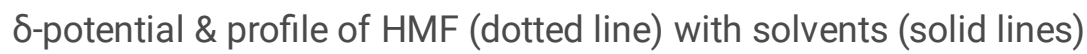

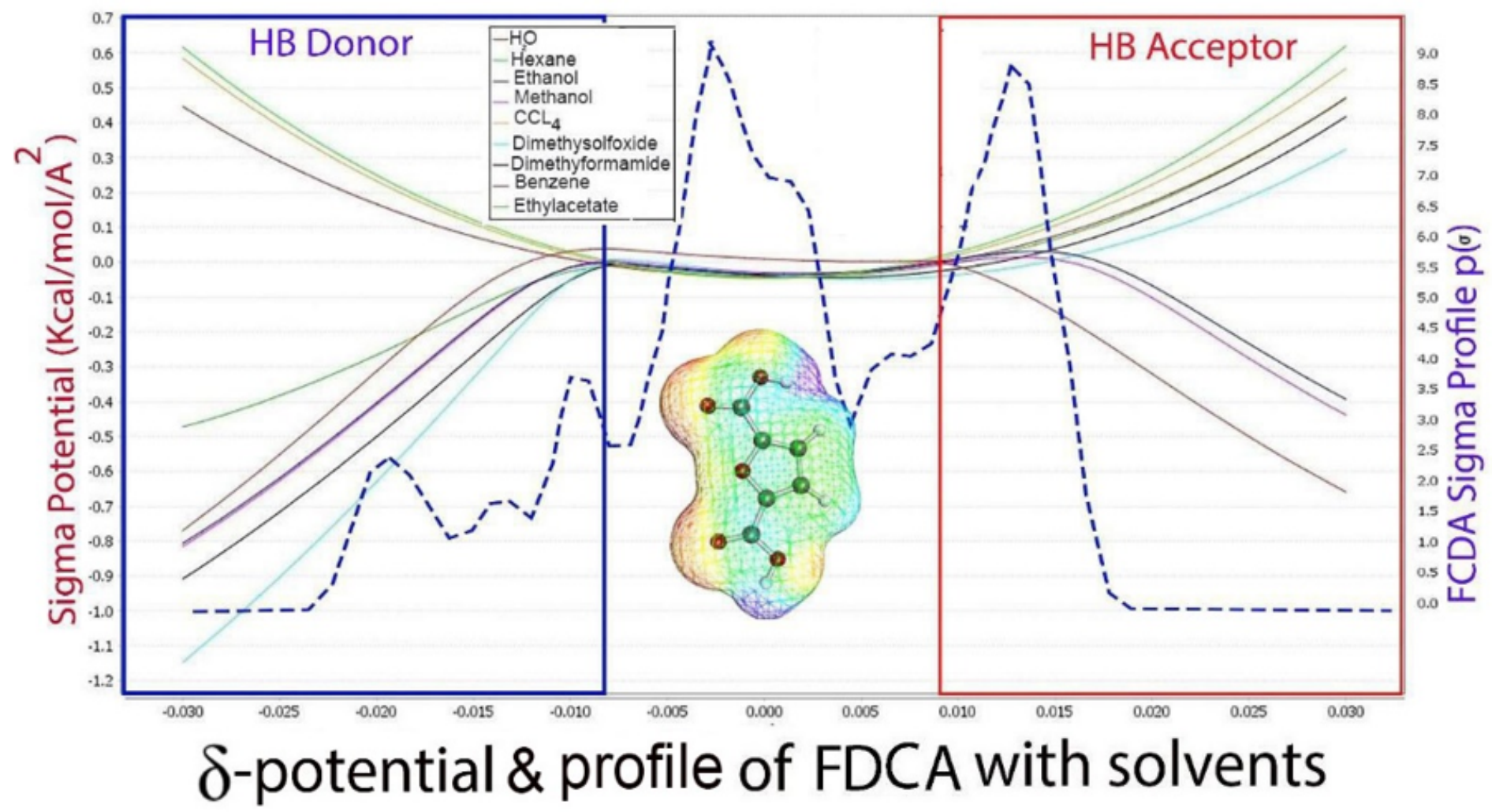


Figure 7

ס-potential \& profile of FDCA with solvents 